\title{
Association of fast food consumption with energy intake, diet quality, body mass index and the risk of obesity in a representative Mediterranean population
}

\author{
Helmut Schröder*, Montserrat Fito and Maria Isabel Covas on behalf of the REGICOR investigators $\dagger$ \\ Lipids and Cardiovascular Epidemiology Research Unit, Institut Municipal d'Investigació Mèdica, IMIM-Hospital de Mar, \\ Biomedical Research Park - Parc de Recerca Biomèdica de Barcelona - PRBB, c/Doctor Aiguader 88, 08003 Barcelona, Spain
}

(Received 23 February 2007 - Revised 8 May 2007 - Accepted 24 May 2007)

The aim of the present study was to describe the association of fast food consumption with BMI, energy intake and diet quality in a Mediterranean population. The subjects were Spanish men $(n$ 1491) and women ( $n$ 1563) aged 25-74 years who were examined in 1999-2000, in a populationbased cross-sectional survey in northeast Spain (Girona). Dietary intake was assessed using a FFQ that included four typical fast food items. Two dietary-quality indices, the Mediterranean diet score and the healthy eating index, were created. Height and weight were measured. Within the population studied, $10.1 \%$ reported eating fast food at least once per month. Dietary energy intake and energy density were directly associated with frequency of fast food consumption. Multivariate logistic regression analysis adjusted for lifestyle and educational level showed an inverse association of frequency of fast food consumption with meeting the dietary reference intake (DRI) for energy $(P=0 \cdot 001)$. The consumption of fast food more than once per week increased the risk of overall low diet quality $(P<0.001)$. BMI was directly associated with fast food consumption expressed in $\mathrm{g} / \mathrm{d}(P=0.025)$ and in $\mathrm{kJ} / \mathrm{d}(P=0.017)$. The risk of being obese increased with the frequency of fast food consumption $(P=0.046)$. Fast food consumption was associated with higher energy intakes, poor diet quality and higher BMI. The likelihood of not meeting the DRI for energy, and of being obese, increased with the frequency of fast food consumption.

Fast food: Diet quality: Body mass index: Mediterranean diet: Obesity: Energy density

Foods available in fast food outlets (henceforth termed fast food) are becoming an increasingly important part of the diet, particularly in the USA ${ }^{1}$. The number of fast food outlets and sales increased dramatically during the past 30 years in North America $^{2}$ and, to a lesser degree, also in Europe. There are several factors inherent to fast food that might promote energy imbalance ${ }^{3}$. Fast food products are often characterised by their high content of fat and sugars, high palatability, large portion size and high energy density.

In the US population, about one-third of total energy intake is derived from fast food consumption ${ }^{4}$. Furthermore, it often displaces healthier food options, reducing thereby the quality of the whole diet. For these reasons it is not surprising that fast food consumption has been associated with increased risk of excessive weight and low overall diet quality in children, adolescents and adults in the USA ${ }^{1-6}$. A Spanish study reported a direct association of weight gain and fast food consumption (proxy variables including hamburgers, pizza and sausages) in a cohort of university graduates?
The increasing number of individuals with excessive weight is an important health concern in most European countries ${ }^{8}$. The identification of lifestyle variables that are associated with this trend is crucial for health policy. Although there is information on the impact of fast food consumption on lifestyle and BMI in the USA, to the best of our knowledge no population-level scientific evidence on the association of fast food consumption with overall diet quality and anthropometric measures is available for Europe. If the impact of fast food consumption on diet quality and BMI, as seen in the USA, is confirmed in European populations, this evidence is essential for health policy makers in their efforts to combat the growing obesity epidemic.

The aim of the present study was to analyse the association of frequency of fast food consumption with energy intake, meeting dietary reference intakes (DRI) for energy, diet quality as characterised by the Mediterranean diet score (MDS) and healthy eating index (HEI), BMI and obesity in a representative Mediterranean population.

Abbreviations: DRI, dietary reference intake; EER, estimated energy requirement; HEI, healthy eating index; MDS, Mediterranean diet score; PA, physical activity.

* Corresponding author: Dr Helmut Schröder, fax: + 34933160 410, email hschroeder@imim.es

$\dagger$ REGICOR investigators: M. I. Covas, R. Elosua, M. Fitó, M. Gil, J. M. Manresa, J. Marrugat, S. Martín, R. Masiá, A. Pena, G. Pérez, I. Ramió, I. Rolhfs, P. Roset, J. Sala, H. Schröder, M. Sentí and J. Vila. 


\section{Subjects and methods}

\section{Study population}

The methods used in the population-based cross-sectional survey of cardiovascular risk conducted in Girona (Spain) in 2000 are identical to those of the cross-sectional survey of 1995, described in detail elsewhere ${ }^{9}$. Using the 1996 census, 6000 subjects aged 25-74 years were randomly selected from the general population of Girona. After excluding census errors, 4359 eligible subjects remained, of whom 3058 agreed to participate in the present study from 1999 to 2000. Dietary data were available from 2930 participants. All participants signed an informed consent form to allow the acquisition of biological samples for analyses and the storage of their personal data in a computer database. The protocol was approved by an ethics committee and the results of the examination were sent to all participants.

\section{Measurement of diet and fast food consumption}

The FFQ comprised 165 items, including foods, alcohol, and non-alcoholic beverages ${ }^{10}$. For each food item, participants were asked to indicate their usual consumption from the nine frequency categories, ranging from never or less than once per month to six or more times per d. The FFQ did not include standard questions on portion size but rather indicated specific medium servings, defined by natural (for example, one orange, one slice of bread) or household units (for example, one spoon, one cup, one glass). The FFQ included frequency of consumption of the following fast food products: hamburger (McDonalds or similar); cheeseburger (McDonalds or similar); BigMac (McDonalds or similar); French fries (McDonalds or similar). Energy consumption and nutrient intake were calculated using the Medisystem 2000 software (Conaycite, Madrid, Spain).

\section{Measurement of diet quality and energy requirement}

We computed two indices, namely MDS and HEI, to calculate the overall diet quality. With the exception of red wine, the MDS was calculated according to the tertile distribution of energy-adjusted food consumption. The lowest tertile was coded as 1 , medium as 2 , and highest as 3 , for cereals, fruits, vegetables, legumes, fish, olive oil, and nuts. The highest tertile was coded as 1 , medium as 2 , and lowest as 3 for meat and dairy products. Red wine consumption was computed as alcohol intake proceeding from red wine $(0 \mathrm{~g}$ and more than $20 \mathrm{~g}$ alcohol $=1$, and up to $20 \mathrm{~g}$ alcohol $=3$ ). The values of distribution of all dietary components were calculated. The resulting MDS ranged from 10 to 30 .

The percentages of energy provided through typical and non-typical foods of a healthy Mediterranean reference diet were calculated. The HEI consists of ten equally weighted components constituting an index of 0 to 100 points $^{11}$. Each of the components represents different dietary recommendations of the food guide pyramid ${ }^{12}$ and the 1990 Dietary Guidelines for Americans ${ }^{13}$. Components 1-5 (grains, vegetables, fruits, meats, and milk) were calculated by comparing the number of servings reported for these five food groups with the recommended number of servings according to age and sex. Each component was scored proportionally from 0 to 10. An energy-adjusted food intake was not used because such an adjustment is not included in the HEI scoring system ${ }^{11}$. Components 6-9 quantify the adherence to recommended dietary intakes of total fat (percentage of energy intake), saturated fat (percentage of energy intake), cholesterol, and $\mathrm{Na}$. We also quantified the variety component differently, using McCullough et al. ${ }^{14,15}$. Subjects were grouped into eleven equal quantiles according to the number of unique foods consumed per month, and the groups were assigned scores of $0-10$.

The estimated energy requirement (EER) was calculated to estimate the prevalence of inadequate energy intake ${ }^{16}$ in the study population. The equation to predict EER includes age, weight, height and physical activity (PA):

$$
\begin{aligned}
\text { EER for men }= & 662-9.53 \times \text { age }(\text { years })+P A \times(15.91 \\
\times & \text { weight }(\mathrm{kg})+539.6 \times \text { height }(\mathrm{m})) ; \\
\text { EER for women }= & 354-6.91 \times \text { age }(\text { years })+\mathrm{PA} \times(9.36 \\
& \times \text { weight }(\mathrm{kg})+726 \times \text { height }(\mathrm{m})) .
\end{aligned}
$$

The equation included four different PA levels: sedentary (PA level $\geq 1$ to $<1.4$ ); low active (PA level $\geq 1.4$ to $<1.6$ ); active (PA level $\geq 1.6$ to $<1.9$ ); very active (PA level $\geq 1.9$ to $<2.5)$ ). The corresponding PA value for sedentary, low active, active and very active PA level is $1 \cdot 0,1 \cdot 12$, $1 \cdot 27$, and 1.45 , respectively.

The PA level in the present population was calculated through the corresponding walking equivalence. DRI for energy was defined as the EER that corresponds to a normal BMI $\left(18.5-24.9 \mathrm{~kg} / \mathrm{m}^{2}\right)^{16}$.

\section{Measurement of non-dietary variables}

Information on demographic and socio-economic variables, medical history, diet, and lifestyle factors including tobacco smoking and alcohol consumption was obtained through structured standard questionnaires administered by trained personnel.

Leisure-time PA was measured by the Minnesota leisuretime physical activity questionnaire, also administered by a trained interviewer. This questionnaire has been previously validated for Spanish men and women ${ }^{17,18}$.

\section{Anthropometric measurements}

A precision scale of easy calibration was used for weight measurement. Readings were rounded to $200 \mathrm{~g}$. Individuals wore underwear. Height was measured in the standing position and measurements rounded to $5 \mathrm{~mm}$. BMI was determined as weight divided by height squared $\left(\mathrm{kg} / \mathrm{m}^{2}\right)$.

\section{Statistical analysis}

The prevalence of fast food consumption was similar in both sexes for average age and also stratified for age groups (25-34 years, 35-44 years, 45-54 years, 55-64 years and 65-74 years). Data are presented with means or proportions. Student's $t$ test and the Mann-Whitney $U$ test were used in 
the univariate analysis for continuous normally and not normally distributed variables, respectively. Logistic regression analysis (PROC LOGISTIC procedure of SAS, version 9.1; SAS Institute Inc., Cary, NC, USA) was performed to determine significance of proportions. General linear modelling procedures (PROC GLM procedure of SAS, version 9.1; SAS Institute Inc.) were used to estimate energy consumption, energy density and food intake (dependent variables) according to the frequency of fast food consumption (fixed factor).

Multiple linear regression models were fitted (PROC REG procedure of SAS, version 9.1; SAS Institute Inc.) to determine the association of BMI with the amount $(\mathrm{g} / \mathrm{d})$ and energy content $(\mathrm{kJ} / \mathrm{d})$ of fast food consumption.

Multiple logistic regression analysis (PROC LOGISTIC procedure of SAS, version 9.1; SAS Institute Inc.) was used to analyse the relationship of recommended energy intake with frequency of fast food consumption as well as the risk of being obese based on frequency of fast food consumption. Differences were considered significant if $P<0 \cdot 05$.

\section{Results}

Of the study population, $10.1 \%$ reported eating fast food at least once per month $(6.3 \%$ once per month, $2.7 \%$ once per week, and $1.1 \%$ more than once per week). Fast food consumption decreased significantly $(P<0.001)$ with increasing age.

Fast food consumers were more likely to be younger, smokers, and more highly educated than non-fast food consumers. They also reported higher energy intake and were more likely to exceed the recommended energy intake (Table 1).

Table 2 presents energy intake and the consumption of foods other than fast food according to the frequency of fast food consumption. Total energy intake and energy density increased across the frequency distribution of fast food consumption. In contrast, the water content of ingested foods and the consumption of fruits and olive oil were inversely associated with increasing frequency of fast food consumption.

Multiple logistical regression analysis revealed an inverse association between diet quality, characterised by the score for adherence to a traditional Mediterranean diet (MDS) and HEI score, and frequency of fast food consumption (Table 3). As shown in Table 3, participants with the highest frequency (more than once per week) of eating a product from a fast food outlet showed the lowest adherence to the MDS (OR 4.30 (95\% CI 1.82, 10.05)) and HEI (OR 3.91 (95\% CI $1.69,9.02)$ ).

Additionally, participants reporting the highest frequency of fast food consumption ( $>$ once per week) showed a significantly higher risk (OR 3.30 (95\% CI 1.52, 7.14)) of failing to meet the DRI for energy consumption as compared with those who consumed no fast foods (Fig. 1).

A multiple linear regression model was fitted to analyse the association of BMI with the amount $(\mathrm{g})$ and energy content $(\mathrm{kJ})$ of fast food consumption (Table 4). BMI increased with the amount $(\mathrm{g} / \mathrm{d})$ of fast food consumption. Because the energy content differed among the fast food items of the FFQ $(\mathrm{kJ} / \mathrm{d})$, we also present data on the relationship between energy intake provided through fast food consumption and BMI. The magnitude of this association was slightly stronger $(P=0.017)$ than that observed for fast food consumption expressed in $\mathrm{g} / \mathrm{d}(P=0.025)$ (Table 4$)$.

Furthermore, there was a significant direct relationship $(P=0.046)$ between obesity and frequency of fast food consumption (Fig. 2). The consumption of a product from a fast food outlet more than once per week increased the risk of being obese by $129 \%(P=0.057)$ in comparison with no consumption.

Table 1. General characteristics of the study population according to the classification of fast food consumption (Percentage of subjects and $95 \%$ confidence intervals)

\begin{tabular}{|c|c|c|c|c|c|}
\hline & \multicolumn{2}{|c|}{$\begin{array}{l}\text { Non- fast food consumers } \\
\text { ( } \text { ( 2577) }\end{array}$} & \multicolumn{2}{|c|}{ Fast food consumers ( $n$ 290) } & \multirow[b]{2}{*}{$P^{*}$} \\
\hline & $\%$ & $95 \% \mathrm{Cl}$ & $\%$ & $95 \% \mathrm{Cl}$ & \\
\hline Men & 89.0 & $87.4,90 \cdot 6$ & $11 \cdot 0$ & $9 \cdot 4,10 \cdot 8$ & \\
\hline Women & 90.7 & $89 \cdot 2,92 \cdot 2$ & $9 \cdot 3$ & $7 \cdot 8,10 \cdot 8$ & 0.128 \\
\hline \multicolumn{6}{|l|}{ Age (years) } \\
\hline Mean & \multicolumn{2}{|c|}{$51 \cdot 1$} & \multicolumn{2}{|c|}{$42 \cdot 6$} & $<0.001$ \\
\hline SD & \multicolumn{2}{|c|}{$13 \cdot 3$} & \multicolumn{2}{|c|}{$13 \cdot 0$} & \\
\hline Educational level (university degree) & 11.8 & $10 \cdot 5,13 \cdot 0$ & $15 \cdot 9$ & $11 \cdot 7,20 \cdot 1$ & 0.036 \\
\hline \multicolumn{6}{|l|}{ LTPA $(M E T) \cdot(\mathrm{min} / \mathrm{d})$} \\
\hline Mean & \multicolumn{2}{|c|}{345.5} & \multicolumn{2}{|c|}{352.4} & 0.681 \\
\hline SD & \multicolumn{2}{|c|}{$356 \cdot 8$} & \multicolumn{2}{|c|}{$344 \cdot 2$} & \\
\hline Smokers & 22.6 & $21 \cdot 0,24 \cdot 2$ & $30 \cdot 6$ & $25 \cdot 3,36 \cdot 0$ & 0.005 \\
\hline Alcohol consumption (g/d) & & & & & 0.369 \\
\hline Mean & \multicolumn{2}{|c|}{11.5} & \multicolumn{2}{|c|}{$12 \cdot 1$} & \\
\hline SD & \multicolumn{2}{|c|}{$17 \cdot 2$} & \multicolumn{2}{|c|}{$19 \cdot 8$} & \\
\hline \multicolumn{6}{|l|}{ Total energy intake (MJ/d) } \\
\hline Mean & \multirow{2}{*}{\multicolumn{2}{|c|}{$\begin{array}{c}10 \cdot 9 \\
3.6\end{array}$}} & \multirow{2}{*}{\multicolumn{2}{|c|}{$\begin{array}{c}12 \cdot 5 \\
4 \cdot 2\end{array}$}} & $<0.001$ \\
\hline SD & & & & & \\
\hline Meeting recommended energy intake & 63.5 & $61 \cdot 6,65 \cdot 4$ & 51.9 & $46 \cdot 1,57 \cdot 8$ & $<0.001$ \\
\hline
\end{tabular}

LTPA, leisure-time physical activity; MET, metabolic equivalent.

* Significance for $\mathrm{P}$ between fast food consumers and non-fast food consumers by Student's $t$ test (continuous variables) and logistical regression analysis (categorical variables). 


\section{Discussion}

The main finding of the present study was that the frequency of fast food consumption was inversely associated with total diet quality and DRI for energy. Furthermore, frequent fast food consumption was directly related to BMI and the risk of obesity. These findings suggest a wake-up call for a region heralded for its healthy diet.

Changes in food supplies have been observed in Mediterranean countries during the last four decades ${ }^{19}$. European Mediterranean countries in particular have undergone a shift from the traditional Mediterranean diet towards a more Westernstyle diet. Concomitant with this trend, prevalence rates of overweight and obesity have considerably increased in these countries ${ }^{8}$. Identifying potentially modifiable contributors to the obesity epidemic is an important public health concern.

In the Continuing Survey of Food Intakes by Individuals (CSFII 1994-6), 26.5\% of adults reported eating fast food in the USA ${ }^{2}$. A comparative study of fast food consumption among various countries in 2004 revealed that $34 \%$ of North Americans ate at take-away restaurants at least once per week, in contrast to only $8 \%$ of Europeans ${ }^{20}$. In the present population, $10.1 \%$ reported fast food consumption; however, only $1.1 \%$ consumed fast food at least once per week. Therefore, it is not surprising that, in general, energy consumption from fast food, both in absolute amounts and in percentage of total energy intake, differed considerably between the USA and the present Mediterranean population. Fast food consumption provided about one-third of the day's energy in the $\mathrm{USA}^{2}$ in comparison to only $1.9 \%$ in the present study population. However, the significance of the findings related to those in the present study who do consume fast food items is sobering.

Scientific evidence indicates that fast food consumption is associated with higher total energy intakes ${ }^{1}$. Our findings support this conclusion. When fast food was excluded from our analysis, total energy intake in the present population was $1.4 \mathrm{MJ} / \mathrm{d}$ higher in fast food consumers than in non-consumers. Furthermore, energy intake from non-fast food sources and from fast food significantly increased with the frequency of fast food consumption. Participants who reported eating fast food more than once per week had a $2.5 \mathrm{MJ} / \mathrm{d}$ higher total (fast food included) energy intake.

Several studies in the USA have associated fast food consumption with poor food choices ${ }^{21,22}$. In the present study we were interested in the impact of fast food consumption on total diet quality. In recent years, a holistic approach based on food consumption (dietary pattern analysis) was developed to quantify the overall quality of a diet ${ }^{23}$. We created two composite indices of dietary behaviours, combining a large amount of information about dietary behaviours into a single indicator of diet quality. Although based on different concepts, the MDS and the HEI are measurement tools that rank subjects according to their adherence to a healthy $\operatorname{diet}^{11,24}$. Most importantly, high adherence to these diet quality indices has been associated with favourable health outcomes $^{25-27}$.

In the present population, it is a serious concern that diet quality was lower for those with higher fast food frequency compared with their peers with lower fast food frequency. The relative risk of having poor diet quality, characterised 
Table 3. Adherence to the Mediterranean diet score (MDS) and healthy eating index (HEI) according to the frequency of fast food consumption ${ }^{\star}$ (Odds ratios and $95 \%$ confidence intervals)

\begin{tabular}{|c|c|c|c|c|c|}
\hline \multirow[b]{2}{*}{ Fast food consumption } & \multirow[b]{2}{*}{$n$} & \multicolumn{2}{|c|}{ MDS† } & \multicolumn{2}{|c|}{ HEl† } \\
\hline & & OR & $95 \% \mathrm{Cl}$ & OR & $95 \% \mathrm{Cl}$ \\
\hline None or less than once per month & 2547 & 1 & Reference & 1 & Reference \\
\hline One to three times per month & 181 & 1.26 & $0.90,1.64$ & 1.31 & $0.93,1.85$ \\
\hline Once per week & 77 & 1.89 & $1.18,3.03$ & 2.95 & $1.77,4.90$ \\
\hline More than once per week & 30 & 4.30 & $1.82,10.05$ & 3.91 & $1.69,9.02$ \\
\hline Linear $P$ for trend & & $<0.001$ & & $<0.001$ & \\
\hline
\end{tabular}

* Lowest tertile of the Mediterranean diet score and the healthy eating index.

†Adjusted for sex, age, energy consumption from non-fast food sources, smoking status and leisure-time physical activity.

through low adherence to the MDS and HEI, was 330 and $291 \%$ higher, respectively, in the $1.1 \%$ of participants who reported eating fast food more than once per week. Since we are not aware of other studies that compare diet quality measured by diet indices with the frequency of fast food consumption, we cannot directly compare our findings. However, other studies have reported lower intakes of healthy foods, such as vegetables, fruits, and legumes, and a less favourable overall nutrient intake profile among fast food consumers in comparison with non-fast food consumers ${ }^{1,6,21,22}$.

A prolonged positive energy imbalance is the driving force of the obesity epidemic that is currently drawing media attention. Increasing energy intake with a concurrent decrease of PA increases the risk of weight gain. In the present study, fast food consumers reported higher energy intakes, excluding energy provided by fast food, than non-consumers. The inability to compensate for the energy in fast food had previously been shown in overweight adolescents ${ }^{28}$. Therefore, it is not surprising that failing to meet the DRI for energy was more frequent among adult consumers of fast food as compared with non-fast food consumers in the present study.

One might speculate that the extra energy provided by fast food $(310$ and $761 \mathrm{~kJ} / \mathrm{d}$ corresponding to the consumption of

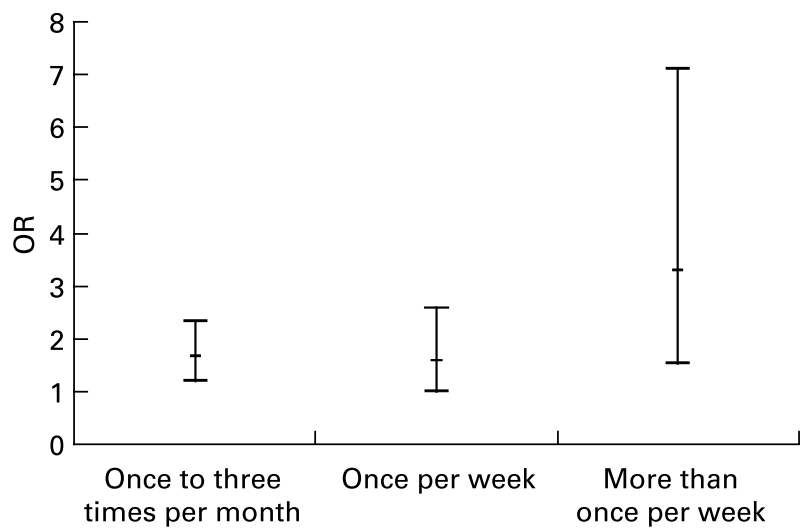

Fast food consumption

Fig. 1. Risk of failing to meet dietary reference intake for energy consumption in relation to frequency of fast food consumption. Values are OR, with $95 \%$ $\mathrm{Cl}$ represented by vertical bars. The reference category is no consumption or less than once per month. The OR are adjusted for educational status, smoking, alcohol consumption and leisure-time physical activity. There is a significant linear trend $(P<0.001)$. fast food once and more than once per week, respectively) in the present population is too low to make a significant contribution to energy imbalance and consequently to an increase in BMI. In a theoretical model, however, it has been estimated that a daily surplus of $66 \mathrm{~kJ}$ leads to $1 \mathrm{~kg}$ per year weight gain $^{29}$.

Table 4. Association between body mass index and fast food consumption*

(Regression coefficients and $95 \%$ confidence intervals)

\begin{tabular}{lccc}
\hline & \multicolumn{3}{c}{ BMI $\left(\mathrm{kg} / \mathrm{m}^{2}\right)$} \\
\cline { 2 - 4 } & $\begin{array}{c}\text { Regression } \\
\text { coefficient }\end{array}$ & $95 \% \mathrm{Cl}$ & $P$ \\
\hline $\begin{array}{c}\text { Fast food } \\
\text { consumption }(\mathrm{g} / \mathrm{d}) \dagger\end{array}$ & 1.760 & $0.223,3.298$ & 0.025 \\
$\begin{array}{c}\text { Fast food } \\
\text { consumption }(\mathrm{kJ} / \mathrm{d}) \dagger\end{array}$ & 1.643 & $0.297,2.99$ & 0.017 \\
\hline
\end{tabular}

${ }^{*}$ Adjusted for sex, age, educational status, leisure-time physical activity, energy consumption of non-fast food sources, smoking status and alcohol drinking. † Variable was log transformed.

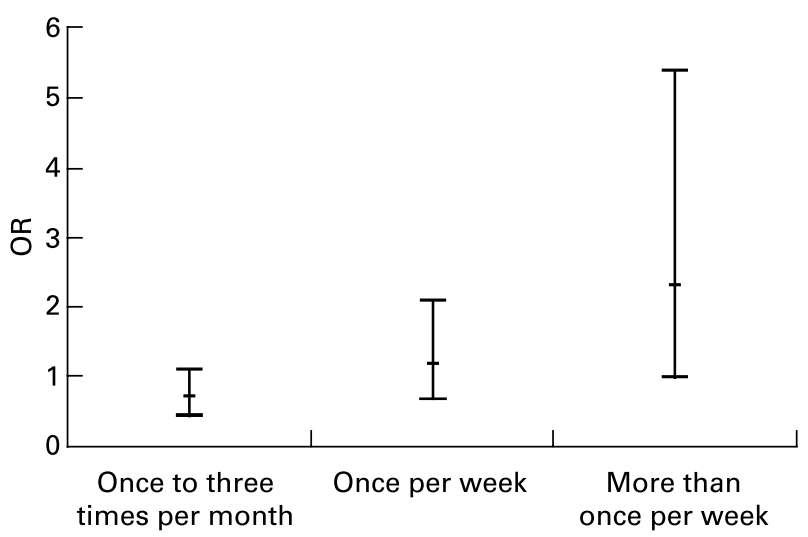

Fast food consumption

Fig. 2. Risk of obesity according to frequency of fast food consumption. Values are OR, with $95 \% \mathrm{Cl}$ represented by vertical bars. The reference category is no fast food consumption. The OR are adjusted for age, sex, leisure-time physical activity, educational status, smoking, alcohol consumption and energy intake from non-fast food sources. There is a significant linear trend $(P<0.046)$. 
In the present population, higher BMI was associated with increasing consumption of fast food. This association was not attenuated after controlling for other lifestyle and socioeconomic confounders. Furthermore, obesity risk increased with the frequency of fast food consumption. These findings agree with previous results concerning fast food consumption and the obesity epidemic in the USA ${ }^{4,5}$. It is of interest to note that the energy density of the diet increased with fast food consumption. Energy-dense diets have been associated with an increasing risk of obesity ${ }^{30,31}$ due to the effects on appetite and food intake regulation ${ }^{32}$ of these diets.

A limitation of the present study's cross-sectional design is that causality among the variables studied cannot be drawn. Furthermore, fast food outlets offer many more products than the four items listed in the FFQ. Although French fries and hamburgers, the products most sold by fast food industry leaders ${ }^{21}$, were included in the questionnaire, a possible underestimation of fast food consumption in the present population cannot be excluded (i.e. participants were not asked whether they ate fast food, but whether they ate four specific fast food items).

In conclusion, energy intake and the prevalence of subjects not meeting the DRI for energy consumption increased with the frequency of fast food consumption; overall diet quality decreased. Higher BMI and increased risk of obesity were related to the reported frequency of fast food consumption. Our findings underline the need for continued monitoring, research and analysis, and the attention of public health agencies to fast food consumption among the European Mediterranean population.

\section{Acknowledgements}

We appreciate the English revision made by Dr Elaine M. Lilly (Writers First Aid). The study was supported by grant 2FD097-0297-CO2-01 from Fondo Europeo de Desarrollo Regional (FEDER) and in part by the Ministerio de Sanidad y Consumo, Instituto de Salud Carlos III, Red HERACLES RD06/0009, CIBER - Epidemiología y salud pública CB06/ 02/0029, CIBER - Fisiopatología de la obesidad y nutrición CB06/03/0028, and Fondo de Invesigación Sanitaria ISCIII CP 03/00 115 .

\section{References}

1. Paeratakul S, Ferdinand DP, Champagne CM, Ryan DH \& Bray GA (2003) Fast-food consumption among US adults and children: dietary and nutrient intake profile. J Am Diet Assoc 103, $1332-1338$.

2. Bowman SA \& Vinyard BT (2004) Fast food consumption of U.S. adults: impact on energy and nutrient intakes and overweight status. J Am Coll Nutr 23, 163-168.

3. Isganaitis E \& Lustig RH (2005) Fast food, central nervous system insulin resistance, and obesity. Arterioscler Thromb Vasc Biol 25, 2451-2462.

4. Bowman SA, Gortmaker SL, Ebbeling CB, Pereira MA \& Ludwig DS (2004) Effects of fast-food consumption on energy intake and diet quality among children in a national household survey. Pediatrics 113, 112-118.

5. Pereira MA, Kartashov AI, Ebbeling CB, Van Horn L, Slattery ML, Jacobs DR Jr \& Ludwig DS (2005) Fast-food habits, weight gain, and insulin resistance (the CARDIA study): 15-year prospective analysis. Lancet 365, 36-42.

6. Schmidt M, Affenito SG, Striegel-Moore R, Khoury PR, Barton B, Crawford P, Kronsberg S, Schreiber G, Obarzanek E \& Daniels S (2005) Fast-food intake and diet quality in black and white girls: the National Heart, Lung, and Blood Institute Growth and Health Study. Arch Pediatr Adolesc Med 159, 626-631.

7. Bes-Rastrollo M, Sanchez-Villegas A, Gomez-Gracia E, Martinez JA, Pajares RM \& Martinez-Gonzalez MA (2006) Predictors of weight gain in a Mediterranean cohort: the Seguimiento Universidad de Navarra Study 1. Am J Clin Nutr 83, $362-370$.

8. Seidell JC (2005) Epidemiology of obesity. Semin Vasc Med 5, 3-14.

9. Masia R, Pena A, Marrugat J, Sala J, Vila J, Pavesi M, Covas M, Aubo C \& Elosua R (1998) High prevalence of cardiovascular risk factors in Gerona, Spain, a province with low myocardial infarction incidence. REGICOR Investigators. $J$ Epidemiol Community Health 52, 707-715.

10. Schroder H, Covas MI, Marrugat J, Vila J, Pena A, Alcantara M \& Masia R (2001) Use of a three-day estimated food record, a 72-hour recall and a food-frequency questionnaire for dietary assessment in a Mediterranean Spanish population. Clin Nutr 20, 429-437.

11. Kennedy ET, Ohls J, Carlson S \& Fleming K (1995) The healthy eating index: design and applications. J Am Diet Assoc 95, 1103-1108.

12. United States Department of Agriculture (1992) The Food Guide Pyramid. Hyattsville, MD: Human Nutrition Information Service.

13. Nutrition and Your Health: Dietary Guidelines for Americans, 3rd edn. Washington, DC: US Department of Health and Human Services, 1990. Home and Garden Bulletin No. 232.

14. McCullough ML, Feskanich D, Rimm EB, Giovannucci EL, Ascherio A, Variyam JN, Spiegelman D, Stampfer MJ \& Willett WC (2000) Adherence to the dietary guidelines for Americans and risk of major chronic disease in men. Am J Clin Nutr 72, 1223-1231.

15. McCullough ML, Feskanich D, Stampfer MJ, Rosner BA, Hu FB, Hunter DJ, Variyam JN, Colditz GA \& Willett WC (2000) Adherence to the dietary guidelines for Americans and risk of major chronic disease in women. Am J Clin Nutr 72, $1214-1222$.

16. Standing Committee on the Scientific Evaluation of Dietary Reference Intakes, Food and Nutrition Board, Institute of Medicine (2002) Dietary Reference Intakes for Energy, Carbohydrate, Fiber, Fat, Fatty Acids, Cholesterol, Protein, and Amino Acids. Washington, DC: National Academy Press.

17. Elosua R, Garcia M, Aguilar A, Molina L, Covas MI \& Marrugat J (2000) Validation of the Minnesota leisure time physical activity questionnaire in Spanish Women. Investigators of the MARATDON group. Med Sci Sports Exerc 32, 1431-1437.

18. Elosua R, Marrugat J, Molina L, Pons S \& Pujol E (1994) Validation of the Minnesota leisure time physical activity questionnaire in Spanish men. The MARATHOM investigators. Am J Epidemiol 139, 1197-1209.

19. Garcia-Closas R, Berenguer A \& Gonzalez CA (2006) Changes in food supply in Mediterranean countries from 1961 to 2001. Public Health Nutr 9, 53-60.

20. ACNielsen, Consumer in Europe - Fast Food / Take Away Consumption. http://www.acnielsen.co.uk/pubs/documents/Euro FastFoodDec04.pdf.

21. French SA, Harnack L \& Jeffery RW (2000) Fast food restaurant use among women in the Pound of Prevention study: dietary, behavioral and demographic correlates. Int $J$ Obes Relat Metab Disord 24, 1353-1359. 
22. Satia JA, Galanko JA \& Siega-Riz AM (2004) Eating at fastfood restaurants is associated with dietary intake, demographic, psychosocial and behavioural factors among African Americans in North Carolina. Public Health Nutr 7, 1089-1096.

23. Hu FB (2002) Dietary pattern analysis: a new direction in nutritional epidemiology. Curr Opin Lipidol 13, 3-9.

24. Trichopoulou A, Kouris-Blazos A, Wahlqvist ML, Gnardellis C, Lagiou P, Polychronopoulos E, Vassilakou T, Lipworth L \& Trichopoulos D (1995) Diet and overall survival in elderly people. BMJ 311, 1457-1460.

25. Trichopoulou A, Costacou T, Bamia C \& Trichopoulos D (2003) Adherence to a Mediterranean diet and survival in a Greek population. N Engl J Med 348, 2599-2608.

26. Schroder H, Marrugat J, Vila J, Covas MI \& Elosua R (2004) Adherence to the traditional Mediterranean diet is inversely associated with body mass index and obesity in a Spanish population. J Nutr 134, 3355-3361.

27. Psaltopoulou T, Naska A, Orfanos P, Trichopoulos D, Mountokalakis T \& Trichopoulou A (2004) Olive oil, the Mediterranean diet, and arterial blood pressure: the Greek European
Prospective Investigation into Cancer and Nutrition (EPIC) study. Am J Clin Nutr 80, 1012-1018.

28. Ebbeling CB, Sinclair KB, Pereira MA, Garcia-Lago E, Feldman HA \& Ludwig DS (2004) Compensation for energy intake from fast food among overweight and lean adolescents. JAMA 291, $2828-2833$.

29. Christiansen E, Garby L \& Sorensen TI (2005) Quantitative analysis of the energy requirements for development of obesity. $J$ Theor Biol 234, 99-106.

30. Howarth NC, Murphy SP, Wilkens LR, Hankin JH \& Kolonel LN (2006) Dietary energy density is associated with overweight status among 5 ethnic groups in the multiethnic cohort study. J Nutr 136, 2243-2248.

31. Ledikwe JH, Blanck HM, Kettel KL, Serdula MK, Seymour JD, Tohill BC \& Rolls BJ (2006) Dietary energy density is associated with energy intake and weight status in US adults. Am J Clin Nutr 83, 1362-1368.

32. Prentice AM \& Jebb SA (2003) Fast foods, energy density and obesity: a possible mechanistic link. Obes Rev 4, 187-194. 